\title{
Asymptotics of Divide-And-Conquer Recurrences Via Iterated Function Systems
}

\author{
John C. Kieffer $\|^{\dagger}$ \\ ${ }^{1}$ Dept. of Electrical \& Computer Engr., University of Minnesota, 200 Union St. SE, Minneapolis, MN 55455, USA
}

Let $k \geq 2$ be a fixed integer. Given a bounded sequence of real numbers $\left(a_{n}: n \geq k\right)$, then for any sequence $\left(f_{n}: n \geq 1\right)$ of real numbers satisfying the divide-and-conquer recurrence

$$
f_{n}=(k-\bmod (n, k)) f_{\lfloor n / k\rfloor}+\bmod (n, k) f_{\lceil n / k\rceil}+a_{n}, \quad n \geq k,
$$

there is a unique continuous periodic function $f^{*}: \mathbb{R} \rightarrow \mathbb{R}$ with period 1 such that $f_{n}=n f^{*}\left(\log _{k} n\right)+o(n)$. If $\left(a_{n}\right)$ is periodic with period $k, a_{k}=0$, and the initial conditions $\left(f_{i}: 1 \leq i \leq k-1\right)$ are all zero, we obtain a specific iterated function system $S$, consisting of $k$ continuous functions from $[0,1] \times \mathbb{R}$ into itself, such that the attractor of $S$ is $\left\{\left(x, f^{*}(x)\right): 0 \leq x \leq 1\right\}$. Using the system $S$, an accurate plot of $f^{*}$ can be rapidly obtained.

Keywords: divide-and-conquer recurrences, iterated function systems, IFS attractor, self-affine functions

\section{Introduction}

In the information theory of data structures (Kieffer et al. (2009), Oh and Kieffer (2010)), one is given an infinite ensemble $\mathcal{E}$ of data structures, each structure consisting of a finite graph with data labels on its vertices, and one then employs a divide-and-conquer compression scheme which uniquely encodes the structures in $\mathcal{E}$ into binary codewords. The scheme works as follows. Letting $\mathcal{E}^{*}$ be a subset of $\mathcal{E}$ such that the complement of $\mathcal{E}^{*}$ in $\mathcal{E}$ is finite, each structure $S \in \mathcal{E}^{*}$ is first decomposed into sub-structures $\left\{S_{i}\right\}$ from $\mathcal{E}$, then the $S_{i}$ 's are separately encoded, and finally one builds the codeword for $S$ by concatenating together the codewords for the $S_{i}$ 's, together with an extra codeword which tells how the $S_{i}$ 's are to be put back together to yield $S$. This compression scheme is governed by the divide-and-conquer recurrence

$$
N(S)=\left[\sum_{i} N\left(S_{i}\right)\right]+M(S), \quad S \in \mathcal{E}^{*},
$$

where $N(S)$ (resp. $N\left(S_{i}\right)$ ) is the length of the codeword for $S$ (resp. $S_{i}$ ), and $M(S)$ is the length of the extra codeword. An asymptotic analysis of recurrence $(1)$ is desired, so that one can see how $N(S)$ grows as the number of vertices of $S$ is allowed to grow without bound. In this way, one is led to the analysis of

\footnotetext{
$\dagger$ J. Kieffer's research was supported in part by the NSF Grant CCF-0830457. 
infinitely many different divide-and-conquer recurrences, one for each ensemble $\mathcal{E}$. The analysis of one of these divide-and-conquer recurrences, put forth in Oh and Kieffer (2010), is the subject of the present paper.

Let $k$ be a fixed integer $\geq 2$. For each $n$ in the set $\mathbb{Z}$ of all integers, $n^{(k)}$ denotes the integer in $\{0,1, \cdots, k-1\}$ which is the remainder upon division of $n$ by $k$. (In Matlab, $n^{(k)}$ is denoted $\bmod (n, k)$, but we prefer the less cumbersome notation $n^{(k)}$.) Let $\left(a_{n}: n \geq k\right)$ be a bounded sequence of real numbers. The divide-and-conquer recurrence to be considered is

$$
f_{n}=\left(k-n^{(k)}\right) f_{\lfloor n / k\rfloor}+n^{(k)} f_{\lceil n / k\rceil}+a_{n}, \quad n \geq k .
$$

The $k=2$ subcase of recurrence (2) was treated in Flajolet and Golin (1994), where the analysis was accomplished via analytic combinatorics. Our analysis for general $k$ does not employ analytic combinatorics; it is an open problem whether analytic combinatorics will work for general $k$. Here is our first result, to be proved in a subsequent paper.

Theorem 1 Let $\left(a_{n}: n \geq k\right)$ be an arbitrary bounded sequence of real numbers. Let $f=\left(f_{n}: n \geq 1\right)$ be a sequence of real numbers satisfying recurrence (2). There is a unique continuous periodic function $f^{*}: \mathbb{R} \rightarrow \mathbb{R}$, with period 1 , such that

$$
f_{n}=n f^{*}\left(\log _{k} n\right)+o(n) .
$$

Notation. Theorem 1 establishes a mapping from a sequence space to a function space. Let us make this precise. Let $\left(a_{n}: n \geq k\right)$ be a fixed bounded sequence of real numbers. We let $\mathcal{S}\left[\left(a_{n}\right)\right]$ denote the set of all sequences $\left(f_{n}: n \geq 1\right)$ of real numbers satisfying the recurrence (2). Note that sequence space $\mathcal{S}\left[\left(a_{n}\right)\right]$ is a convex subset of the vector space of all sequences mapping the set of positive integers into the real line. For each sequence $f=\left(f_{n}: n \geq 1\right)$ belonging to sequence space $\mathcal{S}\left[\left(a_{n}\right)\right]$, we call the function $f^{*}: \mathbb{R} \rightarrow \mathbb{R}$ established by Theorem 1 the rate function of $f$. We define $\mathcal{F}\left[\left(a_{n}\right)\right]$ to be the function space consisting of all rate functions of the sequences in $\mathcal{S}\left[\left(a_{n}\right)\right]$. That is,

$$
\mathcal{F}\left[\left(a_{n}\right)\right] \triangleq\left\{f^{*}: f \in \mathcal{S}\left[\left(a_{n}\right)\right]\right\} .
$$

The mapping $f \rightarrow f^{*}$ from the sequence space $\mathcal{S}\left[\left(a_{n}\right)\right]$ onto the function space $\mathcal{F}\left[\left(a_{n}\right)\right]$ is an affine mapping.

The main results to be obtained in this paper, Theorems 2-5, are stated in the rest of this introduction and assume that the driving sequence $\left(a_{n}\right)$ in recurrence 2 is periodic with period $k$. These results enable one to extract from the recurrence (2) an iterated function system which can be used to characterize all of the rate functions in $\mathcal{F}\left[\left(a_{n}\right)\right]$. (We point out that in Dube (1995), a different iterated function system is extracted from a divide-and-conquer recurrence, from which the order of growth of solutions to the recurrence can be obtained, but this iterated function system cannot be used to obtain the results of the present paper.)

The next result shows that the $o(n)$ term in Theorem 1 is a constant in the periodic $\left(a_{n}\right)$ case.

Theorem 2 Let $\left(a_{n}: n \geq k\right)$ be an arbitrary periodic sequence of real numbers, with period $k$. Let $f=\left(f_{n}: n \geq 1\right)$ be any sequence in $\mathcal{S}\left[\left(a_{n}\right)\right]$. Then $f$ is uniquely determined by its rate function $f^{*}$ according to the formula

$$
f_{n}=n f^{*}\left(\log _{k} n\right)-a_{k} /(k-1), \quad n \geq 1 .
$$


Our next result, Theorem 3, will decompose each rate function $f^{*}$ of Theorem 2 as $f^{*}=g+h$, where $g$ is an explicit simple continuous periodic function on the real line with period 1 depending only on $a_{k}$ and the initial conditions $f_{1}, f_{2}, \cdots, f_{k-1}$, and $h: \mathbb{R} \rightarrow \mathbb{R}$ is a fractal-like rate function arising from taking all initial conditions equal to zero. To state Theorem 3, we need some definitions.

Definitions.

- Let $c=\left(c_{1}, c_{2}, \cdots, c_{k}\right)$ be any $k$-tuple of real numbers such that $c_{k}=k c_{1}$. We define $g^{c}: \mathbb{R} \rightarrow \mathbb{R}$ to be the unique continuous periodic function with period 1 given piecewise over the interval $[0,1)$ by

$$
g^{c}(t)=\left[(r+1) k^{-t}-1\right] c_{r}+\left[1-r k^{-t}\right] c_{r+1}, \quad \log _{k} r \leq t<\log _{k}(r+1), \quad r=1, \cdots, k-1 .
$$

For later use, note that

$$
g^{c}\left(\log _{k} i\right)=c_{i} / i, \quad i=1,2, \cdots, k .
$$

- Let $b=\left(b_{n}: n \in \mathbb{Z}\right)$ be a periodic sequence of real numbers with period $k$, satisfying $b_{k}=0$. Let $\left(h_{n}: n \geq 1\right)$ be the sequence in $\mathcal{S}\left[\left(b_{n}: n \geq k\right)\right]$ for which the initial conditions are

$$
h_{i}=0, \quad i=1, \cdots, k-1 .
$$

We define $h^{b}: \mathbb{R} \rightarrow \mathbb{R}$ to be the rate function of $\left(h_{n}\right)$. By Theorem 2 ,

$$
h_{n}=n h^{b}\left(\log _{k} n\right), \quad n \geq 1 .
$$

Theorem 3 Let $\left(a_{n}: n \geq k\right)$ be an arbitrary periodic sequence of real numbers, with period $k$. Let $b=\left(b_{n}: n \in \mathbb{Z}\right)$ be the periodic sequence with period $k$ such that

$$
b_{n}=a_{n}-a_{k}, \quad n \geq k .
$$

Given any sequence $f=\left(f_{n}: n \geq 1\right)$ in $\mathcal{S}\left[\left(a_{n}\right)\right]$, let $c=\left(c_{1}, \cdots, c_{k}\right)$ be the $k$-tuple

$$
c_{i}=f_{i}+a_{k} /(k-1), \quad i=1,2, \cdots, k \text {. }
$$

Then

$$
f^{*}=g^{c}+h^{b} .
$$

Before stating our final two results, Theorems 4 and 5, we state some facts from Falconer (2003) about iterated function systems and the self-affine functions which can arise from such systems. An iterated function system (IFS) on a closed nonempty subset $X$ of a finite-dimensional Euclidean space is a finite nonempty set $\mathcal{T}$ of continuous mappings from $X$ into itself. The attractor of IFS $\mathcal{T}$, if it exists, is the unique nonempty compact subset $S$ of $X$ such that

$$
S=\cup_{T \in \mathcal{T}} T(S)
$$

A mapping $q:[0,1] \rightarrow \mathbb{R}$ is defined to be self-affine if there is an IFS $\mathcal{T}$ on $[0,1] \times \mathbb{R}$ such that

- Each mapping in $\mathcal{T}$ is an affine mapping. 
- The attractor of $\mathcal{T}$ exists and is equal to $\{(x, q(x)): 0 \leq x \leq 1\}$, the graph of $q$.

We present a useful example of an IFS and a self-affine function whose graph is the attractor of the IFS.

Example 1. Let $b=\left(b_{n}: n \in \mathbb{Z}\right)$ be any periodic sequence of real numbers with period $k$ such that $b_{k}=0$. We define $\mathcal{T}_{b}$ to be the IFS $\left\{T_{0}, T_{1}, \cdots, T_{k-1}\right\}$ on $[0,1] \times \mathbb{R}$ such that each mapping $T_{i}$ is the affine mapping

$$
T_{i}(x, y)=k^{-1}\left(x+i, y+b_{i+1} x+b_{i}(1-x)\right), \quad 0 \leq x \leq 1, \quad y \in \mathbb{R} .
$$

We will show later on in the paper that the attractor of $\mathcal{T}_{b}$ exists and is equal to $\left\{\left(x, q_{b}(x)\right): 0 \leq x \leq 1\right\}$, where $q_{b}$ is a continuous function from $[0,1]$ to $\mathbb{R}$ which satisfies $q_{b}(0)=q_{b}(1)=0$. By definition, $q_{b}$ is self-affine. Since $q_{b}(0)=q_{b}(1)$, there is a unique continuous periodic function $Q_{b}: \mathbb{R} \rightarrow \mathbb{R}$, with period 1 , such that

$$
Q_{b}(x)=q_{b}(x), \quad 0 \leq x \leq 1 .
$$

Theorem 4 which follows allows one to obtain the function $h^{b}$ of Theorem 3 by making a simple change of variable on the function $Q_{b}$.

Theorem 4 Let $b=\left(b_{n}: n \in \mathbb{Z}\right)$ be any periodic sequence of real numbers with period $k$ such that $b_{k}=0$. Then

$$
h^{b}(t)=k^{-t} Q_{b}\left(k^{t}\right), \quad 0 \leq t \leq 1 .
$$

Discussion. Let $\left(a_{n}: n \geq k\right)$ be periodic with period $k$, let $f^{*}$ be any rate function in $\mathcal{F}\left[\left(a_{n}\right)\right]$, and let $b=\left(b_{n}: n \in \mathbb{Z}\right)$ be the periodic sequence with period $k$ such that $(5)$ holds. We discuss a method via which the IFS $\mathcal{T}_{b}$ is exploited to quickly yield a highly accurate plot of $f^{*}$ (such a plot can be useful in applications). Let $G\left(q_{b}\right)=\left\{\left(x, q_{b}(x)\right): 0 \leq x \leq 1\right\}$ be the graph of $q_{b}$. Since $q_{b}(0)=0$, the point $(0,0)$ lies in $G\left(q_{b}\right)$. Define $S_{0}=\{(0,0)\}$, and we recursively define the sequence $\left\{S_{n}: n \geq 1\right\}$ of subsets of $[0,1] \times \mathbb{R}$ by

$$
S_{n}=\cup_{T \in \mathcal{T}_{b}} T\left(S_{n-1}\right), \quad n \geq 1 .
$$

Since $G\left(q_{b}\right)$ is the attractor of $\mathcal{T}_{b}$, each set $S_{n}$ is a subset of $G\left(q_{b}\right)$. Thus, each point $(x, y)$ in $S_{n}$ is of the form $\left(x, q_{b}(x)\right)$, a point on the plot of $q_{b}$. Let $\pi\left(S_{n}\right)$ be the set of first coordinates of the points in $S_{n}$. Since $\pi\left(S_{n}\right)=\left\{i / k^{n}: i=0,1, \cdots, k^{n}-1\right\}$, the set $S_{n}$ consists of $k^{n}$ points on the plot of $q_{b}$. Then, using formula (7), it follows that

$$
h^{b}\left(\log _{k}(x+i)\right)=\frac{q_{b}(x)}{x+i}, \quad x \in \pi\left(S_{n}\right) ; i=1, \cdots, k-1,
$$

which determines $(k-1) k^{n}$ points on the plot of $h^{b}$. Adding in the component $g^{c}$ of $f^{*}$ given in Theorem 3 , we have shown how to obtain $(k-1) k^{n}$ points on the plot of $f^{*}$ from the set $S_{n}$. The set of first coordinates of these points is $\left\{\log _{k}(x+i): x \in \pi\left(S_{n}\right) ; i=1, \cdots, k-1\right\}$, which is dense in $[0,1]$. Therefore, if $n$ is sufficiently large, a highly accurate plot of $h^{b}$ over the interval $[0,1]$ quickly results from the IFS method just outlined. In practice, $n$ does not have to be very large for plot accuracy; for example, for $k=3, n=14$ works well. (See Figure 2 of Oh and Kieffer (2010), which contains several rate function plots obtained via the IFS method.)

Let's call the IFS $\mathcal{T}_{b}$ on $[0,1] \times \mathbb{R}$ the "indirect IFS" because its attractor does not directly yield $h^{b}$, but only after a change of variable. Our final result, Theorem 5 , gives us a "direct IFS" on $[0,1] \times \mathbb{R}$, whose attractor directly yields the function $h^{b}$ without having to make a change of variable. 
Definition. For use in Theorem 5, for each $i=0,1, \cdots, k-1$, we define $U_{i}^{*}:[0,1] \rightarrow[0,1]$ to be the continuous mapping

$$
U_{i}^{*}(t) \triangleq \log _{k}\left(\frac{k^{t}+(i+1)(k-1)}{k}\right), 0 \leq t \leq 1 .
$$

Theorem 5 Let $b=\left(b_{n}: n \in \mathbb{Z}\right)$ be any periodic sequence of real numbers with period $k$ such that $b_{k}=0$. For each $i=0,1, \cdots, k-1$, let $U_{i}:[0,1] \times \mathbb{R} \rightarrow[0,1] \times \mathbb{R}$ be the continuous mapping such that for each $r=1, \cdots, k-1$,

$$
U_{i}(t, z)=\left(U_{i}^{*}(t), \frac{k^{t} z+b_{r-i}\left(k^{t}-r\right)+b_{r-i-1}\left(r+1-k^{t}\right)}{k^{t}+(i+1)(k-1)}\right), \quad \log _{k} r \leq t \leq \log _{k}(r+1), \quad z \in \mathbb{R} .
$$

The attractor of the IFS $\left\{U_{0}, U_{1}, \cdots, U_{k-1}\right\}$ exists and is the set $\left\{\left(x, h^{b}(x)\right): 0 \leq x \leq 1\right\}$.

\section{Remarks.}

- Let $S_{0}=\{(0,0)\}$. The recursion

$$
S_{n}=\cup_{i=0}^{k-1} U_{i}\left(S_{n-1}\right), \quad n \geq 1
$$

arising from the IFS $\left\{U_{i}\right\}$ of Theorem 5 directly yields points on the plot of $h^{b}$. However, it is typically quicker to use the indirect IFS $\mathcal{T}_{b}$ to obtain plot points of $h^{b}$ (as discussed earlier), because the functions in the indirect IFS are simpler.

- To keep the paper self-contained, we prove Theorems 2-4 in subsequent sections of this paper without assuming Theorem 1. The proof of Theorem 5 is omitted; the direct IFS of Theorem 5 can be simply obtained via a change of variable to the indirect IFS of Theorem 4.

\section{A Fundamental Lemma}

Lemma 2 presented at the end of this section will prove to be fundamental in our analysis of the recurrence (2).

Definitions. For each $j \geq 1$, let $T_{k}(j)$ be the finite rooted unordered tree of depth $j$ having $k^{j}$ leaf vertices and $k$ children per each non-leaf vertex. For each $n \geq k$, let $\left(j_{n}, r_{n}\right)$ be the pair of integers such that

- $j_{n}$ is the largest positive integer $j$ such that $k^{j} \leq n$.

- $r_{n}$ is the unique integer $r$ in the set $\{1, \cdots, k-1\}$ such that

$$
r k^{j_{n}} \leq n<(r+1) k^{j_{n}}
$$

Fix $n \geq k$. Label each vertex of the tree $T_{k}\left(j_{n}\right)$ with an integer label as follows. Assign the root of $T_{k}\left(j_{n}\right)$ the label $n$. Given that a non-leaf vertex $v$ of $T_{k}\left(j_{n}\right)$ has been labeled with integer $m$, label $k-m^{(k)}$ of the $k$ children of $v$ with the label $\lfloor m / k\rfloor$ and label the remaining children (if any) with the label $1+\lfloor m / k\rfloor$. We denote the resulting labeled tree $T_{k}^{n}$.

Example 2. Let $k=3$ and $n=23$. Then $j_{n}=2$ and $r_{n}=2$ because the inequality (8) is in this case

$$
2\left(3^{2}\right) \leq 23<3\left(3^{2}\right) \text {. }
$$


The labeled tree $T_{3}^{23}$ is of depth 2 . The root label is 23 . The three depth 1 labels are $7,8,8$. The nine depth 2 labels are the leaf labels and these are 2, 2, 3, 2, 3, 3, 2, 3, 3 .

We omit the simple proofs of the following two lemmas.

Lemma 1 For each $n \geq k$, the labeled tree $T_{k}^{n}$ has the following properties.

- P1: The tree is additive, by which we mean that the label on each non-leaf vertex is the sum of the labels on its children.

- P2: For each $0 \leq j \leq j_{n}$, the labels on the depth $j$ vertices belong to the set $\left\{\left\lfloor n / k^{j}\right\rfloor, 1+\left\lfloor n / k^{j}\right\rfloor\right\}$. In particular, the leaf labels (which are the labels at depth $j_{n}$ ), belong to the set $\left\{r_{n}, r_{n}+1\right\}$.

- P3: For each $0 \leq j \leq j_{n}$, exactly $k^{j}\left(1+\left\lfloor n / k^{j}\right\rfloor\right)$ - nof the depth $j$ labels are equal to $\left\lfloor n / k^{j}\right\rfloor$. In particular, exactly $\left(r_{n}+1\right) k^{j_{n}}-n$ of the leaf labels are equal to $r_{n}$.

Lemma 2 Let $\left(a_{n}: n \geq k\right)$ be any sequence of real numbers, and let $\left(f_{n}: n \geq 1\right)$ be a sequence satisfying recurrence (2). Let $n \geq k$ be fixed. For each vertex $v$ of $T_{k}^{n}$, let $L(v)$ be the label on $v$. Let $V_{1}\left(T_{k}^{n}\right)$ be the set of leaf vertices of $T_{k}^{n}$ and let $V_{2}\left(T_{k}^{n}\right)$ be the set of non-leaf vertices. Then

$$
f_{n}=f_{n, 1}+f_{n, 2}
$$

where

$$
\begin{aligned}
& f_{n, 1} \triangleq \sum_{v \in V_{1}\left(T_{k}^{n}\right)} f_{L(v)}=\left[\left(r_{n}+1\right) k^{j_{n}}-n\right] f_{r_{n}}+\left[n-r_{n} k^{j_{n}}\right] f_{r_{n}+1}, \\
& f_{n, 2} \triangleq \sum_{v \in V_{2}\left(T_{k}^{n}\right)} a_{L(v)} .
\end{aligned}
$$

Remarks. Lemma 2 is fundamental for divide-and-conquer recurrence analysis, even for the case when $\left(a_{n}\right)$ is unbounded, not considered in the present paper. Lemma 2 separates out the contribution to the solution sequence due to the driving sequence $\left(a_{n}\right)$ (which yields labels on the non-leaf vertices of $T_{k}^{n}$ which are added up to obtain $f_{n, 2}$ ) from the contribution due to the initial terms $f_{1}, f_{2}, \cdots, f_{k}$ (which yield labels on the leaf vertices of $T_{k}^{n}$ which are added up to obtain $\left.f_{n, 1}\right)$. If we suppose that $\left(a_{n}: n \geq k\right)$ in Lemma 2 is periodic with period $k$ and $a_{k}=0$, it will be clear in Sec. 5 that $f_{n, 1}=n g^{c}\left(\log _{k} n\right)$ and $f_{n, 2}=n h^{b}\left(\log _{k} n\right)$, where $g^{c}, h^{b}$ are the functions in Theorem 3, whereupon the decomposition 97 in Lemma 2 quickly yields the decomposition $f^{*}=g^{c}+h^{b}$ of Theorem 3 .

\section{Homogeneous Recurrence}

In this section, we examine rate functions in the case of the homogeneous divide-and-conquer recurrence 2 in which the $\left(a_{n}\right)$ sequence is the zero sequence. The following result establishes that the rate functions arising in the homogeneous recurrence case are precisely the $g^{c}$ functions defined in the Introduction.

Proposition 1 Let $\left(g_{n}: n \geq 1\right)$ be any sequence of real numbers such that

$$
g_{n}=\left(k-n^{(k)}\right) g_{\lfloor n / k\rfloor}+n^{(k)} g_{\lceil n / k\rceil}, \quad n \geq k .
$$

Let $c=\left(g_{1}, g_{2}, \cdots, g_{k}\right)$. Then

$$
g_{n}=n g^{c}\left(\log _{k} n\right), \quad n \geq 1
$$


Proof: From (4),

$$
g^{c}\left(\log _{k} n\right)=g_{n} / n, \quad n=1, \cdots, k-1,
$$

and so 10 holds for $1 \leq n<k$. Fix $n \geq k$. We show that 10 holds. Let $j \geq 1$ and $r \in\{1, \cdots, k-1\}$ be the integers such that

$$
r k^{j} \leq n<(r+1) k^{j} .
$$

By Lemma 2,

$$
g_{n}=\left[(r+1) k^{j}-n\right] g_{r}+\left[n-r k^{j}\right] g_{r+1} .
$$

Dividing by $n$,

$$
\left.g_{n} / n=\left[(r+1)\left(k^{j} / n\right)-1\right)\right] g_{r}+\left[1-r\left(k^{j} / n\right)\right] g_{r+1} .
$$

Letting $t=\log _{k}\left(n / k^{j}\right)$, and referring to $\sqrt{3}$, we have

$$
g_{n} / n=\left[(r+1) k^{-t}-1\right] g_{r}+\left[1-r k^{-t}\right] g_{r+1}=g^{c}(t) .
$$

Since $g^{c}$ is periodic with period 1 ,

$$
g^{c}(t)=g^{c}\left(\left(\log _{k} n\right)-j\right)=g^{c}\left(\log _{k} n\right) .
$$

Thus, (10) holds.

\section{Rate Functions Arising From Zero Initial Conditions}

Throughout this section, fix $b=\left(b_{n}: n \in \mathbb{Z}\right)$ to be a periodic sequence of real numbers with period $k$ such that $b_{k}=0$. Consider the sequence $\left(h_{n}: n \geq 1\right)$ satisfying the recurrence

$$
h_{n}=\left(k-n^{(k)}\right) h_{\lfloor n / k\rfloor}+n^{(k)} h_{\lceil n / k\rceil}+b_{n}, \quad n \geq k,
$$

subject to the zero initial conditions

$$
h_{i}=0, \quad i=1, \cdots, k-1 .
$$

We defined $h^{b}$ in the Introduction as the rate function of $\left(h_{n}: n \geq 1\right)$, that is, we assumed there is a (necessarily unique) continuous periodic function $h^{b}: \mathbb{R} \rightarrow \mathbb{R}$ with period 1 such that

$$
h_{n}=n h^{b}\left(\log _{k} n\right), \quad n \geq 1 .
$$

In order to keep this paper self-contained, we do not yet assume that $h^{b}$ exists satisfying these properties. It is our task in this section to show that $h^{b}$ exists and to find a useful characterization of $h^{b}$, culminating in a proof of Theorem 4. In order to proceed further, we need the following definitions.

Definitions.

- For each $j \geq 0, \Lambda_{j}(k)$ is defined to be the set of all pairs $(m, n)$ of non-negative integers such that $m+n=k^{j}$, and $\Lambda(k)$ is defined to be the union of the sets $\Lambda_{j}(k)$ over all $j \geq 0$. Note that if $(m, n)$ belongs to the set $\Lambda_{j}(k)$, where $j>0$, then the pairs

$$
(\lfloor m / k\rfloor,\lceil n / k\rceil), \quad(\lceil m / k\rceil,\lfloor n / k\rfloor)
$$


belong to the set $\Lambda_{j-1}(k)$, and we have the vector sum

$$
(m, n)=\left(k-m^{(k)}\right)(\lfloor m / k\rfloor,\lceil n / k\rceil)+m^{(k)}(\lceil m / k\rceil,\lfloor n / k\rfloor) .
$$

Also, note that $\Lambda_{0}(k)$ consists of just the two pairs $(1,0)$ and $(0,1)$.

- $H_{b}: \Lambda(k) \rightarrow \mathbb{R}$ is defined to be the function satisfying

$$
H_{b}(0,1)=H_{b}(1,0)=0,
$$

and the property that if $(m, n) \in \Lambda(k)$ and $m+n>1$, then

$$
H_{b}(m, n)=\left(k-m^{(k)}\right) H_{b}(\lfloor m / k\rfloor,\lceil n / k\rceil)+m^{(k)} H_{b}(\lceil m / k\rceil,\lfloor n / k\rfloor)+b_{m} .
$$

Proposition 2 There is a unique continuous periodic function $H_{b}^{*}: \mathbb{R} \rightarrow \mathbb{R}$, with period 1 , such that

$$
H_{b}(m, n)=(m+n) H_{b}^{*}\left(\frac{m}{m+n}\right), \quad(m, n) \in \Lambda(k) .
$$

Proof Sketch of Proof: $H_{b}^{*}$ is uniquely defined on the set $D=\left\{i / k^{n}: i=0,1, \cdots, k^{n} ; n=0,1,2, \cdots\right\}$ due to the property $H_{b}(k m, k n)=k H_{b}(m, n) . H_{b}^{*}$ is uniformly continuous on $D$ by the argument given in Appendix A of $\operatorname{Kieffer}(2011) . H_{b}^{*}$ then uniquely extends to a continuous function on $[0,1]$ since $D$ is dense in $[0,1]$. Finally, $H_{b}^{*}$ has a periodic extension to the real line because $H_{b}^{*}(0)=H_{b}^{*}(1)=0$.

Remark. Prop. 4 at the end of this section will establish that the function $H_{b}^{*}$ coincides with the function $Q_{b}$ of the Introduction.

Definition. Since $H_{b}^{*}(0)=0$ and $H_{b}^{*}$ is continuous and periodic with period 1 , there exists a unique continuous periodic function $\phi: \mathbb{R} \rightarrow \mathbb{R}$ with period 1 such that

$$
\phi(t)=k^{-t} H_{b}^{*}\left(k^{t}\right), \quad 0 \leq t \leq 1 .
$$

Proposition 3 Let $\left(h_{n}: n \geq 1\right)$ be the sequence satisfying $(11)-(12)$. Then

$$
h_{n}=n \phi\left(\log _{k} n\right), \quad n \geq 1 .
$$

Proof: The first part of the proof is to show that for each $r \in\{1, \cdots, k-1\}$,

$$
H_{b}(m, n)=h_{(r+1) m+r n}, \quad(m, n) \in \Lambda(k) .
$$

The proof is by induction on $m+n$ for $(m, n) \in \Lambda(k)$. If $m+n=1$, then $(m, n)=(1,0)$ or $(m, n)=(0,1)$. If $(m, n)=(1,0)$, then $h_{(r+1) m+r n}=h_{r+1}=0$ and 15$)$ holds because $H_{b}(1,0)=0$. If $(m, n)=(0,1)$, then $h_{(r+1) m+r n}=h_{r}=0$ and $[15$ again holds. For our induction hypothesis, we assume that 15$]$ holds for all $(m, n) \in \Lambda_{j}(k)$, where $j$ is a fixed positive integer. The proof by induction is completed by showing that 15 holds for all $(m, n) \in \Lambda_{j+1}(k)$. Let $(m, n)$ be such a pair. Then

$$
H_{b}(m, n)=\left(k-m^{(k)}\right) H_{b}\left(m_{1}, n_{1}\right)+m^{(k)} H_{b}\left(m_{2}, n_{2}\right)+b_{m},
$$


where

$$
\left(m_{1}, n_{1}\right)=(\lfloor m / k\rfloor,\lceil n / k\rceil), \quad\left(m_{2}, n_{2}\right)=(\lceil m / k\rceil,\lfloor n / k\rfloor) .
$$

For $i=1,2$, we have $\left(m_{i}, n_{i}\right) \in \Lambda_{j}(k)$, whence by the induction hypothesis

$$
H_{b}\left(m_{i}, n_{i}\right)=h_{(r+1) m_{i}+r n_{i}}, \quad i=1,2 .
$$

Let $q=(r+1) m+r n=r k^{j+1}+m$. Then $q$ and $m$ are congruent modulo $k$, and so $q^{(k)}=m^{(k)}$ and $b_{q}=b_{m}$ by the periodicity of the $b$ sequence. It then follows that

$$
\begin{aligned}
h_{q} & =\left(k-q^{(k)}\right) h_{\lfloor q / k\rfloor}+q^{(k)} h_{\lceil q / k\rceil}+b_{q} \\
& =\left(k-m^{(k)}\right) h_{\lfloor q / k\rfloor}+m^{(k)} h_{\lceil q / k\rceil}+b_{m} .
\end{aligned}
$$

We also have

$$
\begin{aligned}
& \lfloor q / k\rfloor=r k^{j}+m_{1}=(r+1) m_{1}+r n_{1}, \\
& \lceil q / k\rceil=r k^{j}+m_{2}=(r+1) m_{2}+r n_{2},
\end{aligned}
$$

and so

$$
\left(k-m^{(k)}\right) h_{\lfloor q / k\rfloor}+m^{(k)} h_{\lceil q / k\rceil}=\left(k-m^{(k)}\right) H_{b}\left(m_{1}, n_{1}\right)+m^{(k)} H_{b}\left(m_{2}, n_{2}\right)
$$

by assumption (17). Adding $b_{m}$ to both sides, we have by 16 and 18 that

$$
H_{b}(m, n)=h_{q}=h_{(r+1) m+r n},
$$

completing the proof of statement (15). We now prove (14). If $1 \leq n<k$, the left side of formula (14) is zero, and the right side is also zero because $\phi\left(\log _{k} n\right)=H_{b}^{*}(n) / n=0$. Let us now establish the formula 14. for a fixed $n \geq k$. We may express $n$ as

$$
n=r k^{j}+m
$$

in which $r, j, m$ are integers satisfying

$$
1 \leq r \leq k-1, \quad j \geq 1, \quad 0 \leq m \leq k^{j} .
$$

By $[15]$ and Proposition 2,

$$
h_{n}=H_{b}\left(m, k^{j}-m\right)=k^{j} H_{b}^{*}\left(m k^{-j}\right) .
$$

Let $t \in[0,1]$ be the real number

$$
t=\log _{k}\left(r+m k^{-j}\right)=\log _{k}\left(n / k^{j}\right) .
$$

Since

$$
m k^{-j}=k^{t}-r, \quad k^{j}=n k^{-t},
$$

and the period of the periodic function $H_{b}^{*}$ is 1 , we have

$$
h_{n}=n k^{-t} H_{b}^{*}\left(k^{t}-r\right)=n k^{-t} H_{b}^{*}\left(k^{t}\right)=n \phi(t) .
$$

But the period of the periodic function $\phi$ is also 1 , and so

$$
h_{n} / n=\phi(t)=\phi\left(\log _{k}\left(n / k^{j}\right)\right)=\phi\left(\left(\log _{k} n\right)-j\right)=\phi\left(\log _{k} n\right) \text {, }
$$

establishing (14). 
Corollary 1 The continuous periodic function $h^{b}: \mathbb{R} \rightarrow \mathbb{R}$ with period 1 satisfying $(13$ ) exists and can be obtained by the change of variable

$$
h^{b}(t)=k^{-t} H_{b}^{*}\left(k^{t}\right), \quad 0 \leq t \leq 1 .
$$

Proof: $h^{b}$ is the function $\phi$ of Proposition 3 .

In view of the preceding Corollary, the establishment of Theorem 4 will be complete once we identify $H_{b}^{*}$ as the periodic function $Q_{b}$ of the Introduction such that $\left\{\left(x, Q_{b}(x)\right): 0 \leq x \leq 1\right\}$ is the attractor of the IFS $\mathcal{T}_{b}$. This will be done in our final result of this section, Proposition 4.

Definition. For each $i=0,1, \cdots, k-1$, we define $T_{i}^{*}:[0,1] \rightarrow[0,1]$ to be the affine function

$$
T_{i}^{*}(x)=k^{-1}(x+i), \quad 0 \leq x \leq 1 .
$$

Each affine mapping $T_{i}$ on $[0,1] \times \mathbb{R}$ in the IFS $\mathcal{T}_{b}=\left\{T_{0}, T_{1}, \cdots, T_{k-1}\right\}$ can be re-expressed as

$$
T_{i}(x, y)=\left(T_{i}^{*}(x), k^{-1}\left[y+b_{i+1} x+b_{i}(1-x)\right]\right), \quad x \in[0,1], y \in \mathbb{R} .
$$

Proposition 4 For each $i=0,1, \cdots, k-1$,

$$
H_{b}^{*}\left(T_{i}^{*}(x)\right)=k^{-1}\left[H_{b}^{*}(x)+b_{i+1} x+b_{i}(1-x)\right], 0 \leq x \leq 1 .
$$

Furthermore, the attractor of IFS $\mathcal{T}_{b}$ exists and is $\left\{\left(x, H_{b}^{*}(x)\right): 0 \leq x \leq 1\right\}$.

Proof: Fix $i \in\{0,1, \cdots, k-1\}$. Let $M_{i}$ be the matrix

$$
M_{i}=\left[\begin{array}{cc}
i+1 & k-i-1 \\
i & k-i
\end{array}\right] .
$$

Since $M_{i}$ has row sums each equal to $k$, it is easy to show that $(m, n) M_{i}$ belongs to the set $\Lambda(k)$ if $(m, n)$ does. The first part of the proof is to establish the identity

$$
H_{b}\left((m, n) M_{i}\right)=H_{b}(m, n)+m b_{i+1}+n b_{i}, \quad(m, n) \in \Lambda(k) .
$$

If $(m, n)=(1,0)$, the right side of 20$)$ is $b_{i+1}$ and by the recurrence 16 , the left side is

$$
H_{b}(i+1, k-i-1)=(k-i-1) H_{b}(0,1)+(i+1) H_{b}(1,0)+b_{i+1}=b_{i+1} .
$$

Thus, 20 holds for $(m, n)=(1,0)$. Similarly, 20 can be seen to hold for $(m, n)=(0,1)$, with both sides being equal to $b_{i}$. The rest of the proof of $(20)$ for all $(m, n) \in \Lambda(k)$ is an induction argument on $m+n$, which we omit because this argument is similar to an induction argument given in the proof of Theorem 2 of Kieffer (2011). In 20, let $\left(m_{i}, n_{i}\right)=(m, n) M_{i}$. Then, by Proposition 2, we may rewrite 20] as

$$
\left(m_{i}+n_{i}\right) H_{b}^{*}\left(m_{i} /\left(m_{i}+n_{i}\right)\right)=(m+n) H_{b}^{*}(m /(m+n))+m b_{i+1}+n b_{i} .
$$

Let $x=m /(m+n)$. Since $m_{i}+n_{i}=k(m+n)$, we obtain

$$
H_{b}^{*}\left(m_{i} /\left(m_{i}+n_{i}\right)\right)=k^{-1}\left[H_{b}^{*}(x)+b_{i+1} x+b_{i}(1-x)\right]
$$


when we divide both sides of (21) by $k(m+n)$. Noting that

$$
m_{i} /\left(m_{i}+n_{i}\right)=[(i+1) m+i n] /[k(m+n)]=k^{-1}[(i+1) x+i(1-x)]=T_{i}^{*}(x),
$$

we see that 19 holds for all $x$ in the set $D=\{m /(m+n):(m, n) \in \Lambda(k)\}$. Since the set $D$ is dense in $[0,1]$ and $H_{b}^{*}$ is continuous, formula $[19)$ holds for all $x \in[0,1]$.

To complete the proof of Prop. 4, letting mapping $q_{b}$ be the restriction of $H_{b}^{*}$ to $[0,1]$, we need to explain why IFS $\mathcal{T}_{b}=\left\{T_{i}: i=0, \cdots, k-1\right\}$ has attractor $S=\left\{\left(x, q_{b}(x)\right): 0 \leq x \leq 1\right\}$. Statement [19] is equivalent to the statement that $\cup_{i} T_{i}(S) \subset S$. We have to obtain the stronger statement $\cup_{i} T_{i}(S)=S$, as well as uniqueness of $S$. We sketch how this can be shown via a perturbation argument. For each $i=0,1, \cdots, k-1$, let $T_{i}^{\epsilon}$ be the mapping on $[0,1] \times \mathbb{R}$ defined by

$$
T_{i}^{\epsilon}(x, y) \triangleq\left(T_{i}^{*}(x), k^{-1}\left[y+\epsilon b_{i+1} x+\epsilon b_{i}(1-x)\right]\right), \quad x \in[0,1], y \in \mathbb{R},
$$

where $\epsilon$ is chosen to be a real number in the interval $(0,1)$ so small that each $T_{i}^{\epsilon}$ is a contraction mapping with respect to the Euclidean metric on $[0,1] \times \mathbb{R}$. The IFS $\mathcal{T}_{b}^{\epsilon}=\left\{T_{i}^{\epsilon}: i=0, \cdots, k-1\right\}$ is one of infinitely many iterated function systems on $[0,1] \times \mathbb{R}$ shown on page 153 of Falconer (2003) to possess attractors which are the graphs of continuous self-affine functions on $[0,1]$. Accordingly, let $q_{b}^{\epsilon}$ be the continuous self-affine function on $[0,1]$ whose graph $\left\{\left(x, q_{b}^{\epsilon}(x)\right): 0 \leq x \leq 1\right\}$ is the attractor $S_{\epsilon}$ of $\mathcal{T}_{b}^{\epsilon}$. By uniqueness of $S_{\epsilon}, q_{b}^{\epsilon}$ is the unique continuous mapping $q$ on $[0,1]$ satisfying the system of $k$ equations

$$
q\left(T_{i}^{*}(x)\right)=k^{-1}\left[q(x)+\epsilon b_{i+1} x+\epsilon b_{i}(1-x)\right], \quad 0 \leq x \leq 1(i=0,1, \cdots, k-1) .
$$

But by (19), this system of equations is satisfied for $q=\epsilon q_{b}$, so we must have $q_{b}^{\epsilon}=\epsilon q_{b}$, and therefore $S_{\epsilon}=\left\{\left(x, \epsilon q_{b}(x)\right): 0 \leq x \leq 1\right\}$. Statement $\cup_{i} T_{i}(S)=S$ then easily follows from the statement $\cup_{i} T_{i}^{\epsilon}\left(S_{\epsilon}\right)=S_{\epsilon}$. The fact that equation $S=\cup_{i} T_{i}(S)$ has a unique (compact) solution $S$ will follow from the fact that equation $S_{\epsilon}=\cup_{i} T_{i}^{\epsilon}\left(S_{\epsilon}\right)$ has a unique (compact) solution $S_{\epsilon}$, via an argument which we omit.

\section{Proof of Theorems 2-3}

The following result implies both Theorems 2 and 3.

Proposition 5 Let $\left(a_{n}: n \geq k\right)$ be a periodic sequence of real numbers with period $k$. Let $\left(f_{n}: n \geq 1\right)$ be any sequence of real numbers satisfying recurrence (2). Let c be the $k$-tuple defined by (6) and let $b=\left(b_{n}: n \in \mathbb{Z}\right)$ be the sequence defined by (5). Then

$$
f_{n}=n\left[g^{c}\left(\log _{k} n\right)+h^{b}\left(\log _{k} n\right)\right]-a_{k} /(k-1), \quad n \geq 1 .
$$

Proof Sketch of Proof: We present the main steps of the proof, omitting the easily verifiable details of carrying out the steps. Let $\left(q_{n}: n \geq 1\right)$ be the sequence

$$
q_{n}=f_{n}+a_{k} /(k-1), \quad n \geq 1
$$

The first step of the proof is to show that $\left(q_{n}\right)$ satisfies the recurrence

$$
q_{n}=\left(k-n^{(k)}\right) q_{\lfloor n / k\rfloor}+n^{(k)} q_{\lceil n / k\rceil}+b_{n}, \quad n \geq k .
$$


Let $\left(q_{n}^{1}: n \geq 1\right)$ be the sequence satisfying the recurrence

$$
q_{n}^{1}=\left(k-n^{(k)}\right) q_{\lfloor n / k\rfloor}^{1}+n^{(k)} q_{\lceil n / k\rceil}^{1}, \quad n \geq k
$$

for which the initial conditions are

$$
q_{i}^{1}=q_{i}, \quad i=1, \cdots, k-1 .
$$

Let $\left(q_{n}^{2}: n \geq 1\right)$ be the sequence satisfying the recurrence

$$
q_{n}^{2}=\left(k-n^{(k)}\right) q_{\lfloor n / k\rfloor}^{2}+n^{(k)} q_{\lceil n / k\rceil}^{2}+b_{n}, \quad n \geq k
$$

with the zero initial conditions

$$
q_{i}^{2}=0, \quad i=1, \cdots, k-1 .
$$

The second step of the proof is to show that

$$
q_{n}=q_{n}^{1}+q_{n}^{2}, \quad n \geq 1 .
$$

By Propositions 1 and 3,

$$
q_{n}^{1}=n g^{c}\left(\log _{k} n\right), \quad q_{n}^{2}=n h^{b}\left(\log _{k} n\right), \quad n \geq 1 .
$$

Therefore,

$$
q_{n}=q_{n}^{1}+q_{n}^{2}=n\left[g^{c}\left(\log _{k} n\right)+h^{b}\left(\log _{k} n\right)\right], \quad n \geq 1 .
$$

Substituting $q_{n}=f_{n}+a_{k} /(k-1)$, we obtain 22.

\section{References}

S. Dube. Using fractal geometry for solving divide-and-conquer recurrences. J. Austral. Math. Soc. Ser. $B, 37: 145-171,1995$.

K. Falconer. Fractal Geometry. John Wiley \& Sons, Chichester, England, 2003.

P. Flajolet and M. Golin. Mellin transforms and asymptotics. Acta Informatica, 31:673-696, 1994.

J. Kieffer. A catalog of self-affine hierarchical entropy functions. Algorithms (Basel), 4:307-333, 2011.

J. Kieffer, E.-H. Yang, and W. Szpankowski. Structural complexity of random binary trees. In Proceedings of the IEEE International Symposium on Information Theory, Seoul, Korea, 2009.

S.-Y. Oh and J. Kieffer. Fractal compression rate curves in lossless compression of balanced trees. In Proceedings of the IEEE International Symposium on Information Theory, Austin, Texas, 2010. 